\title{
Les oncogènes cellulaires, clés de la cancérogenèse
}

Certains virus cancérogènes tirent leur pouvoir de l'intégration de gènes cellulaires d'organismes supérieurs dans leur structure. La réintroduction de ces séquences dans de nouvelles cellules infectées provoque un dérèglement de plusieurs mécanismes du cycle cellulaire. Cette découverte ouvre la voie à une théorie unificatrice du cancer.

\section{Dominique Stéhelin}

Directeur du Laboratoire d'oncologie moléculaire. Inserm U I86.

Une partie de cet article est extraite de Théorie et pratiques thérapeutiques $n^{\circ} 44,1984$.

\section{REFERENCES}

I. Stéhelin D., Guntaka R. V., Varmus H. E., Bishop J. M. Purification of DNA complemenwry to nucleotide sequences required for neoplastic transformation of fibroblasts by ASV. $7 \mathrm{Mol}$ Biol 1976; 101 : 349-65.

2. Stéhelin D., Varmus H. E., Bishop J. M., Vogt P. K. DNA related to the transforming gene(s) of avian sarcoma virus is present in normal avian DNA. Nature 1976; 260: 170-3.

3. Bishop J. M. Cellular oncogenes and retroviruses. Annu Rev Biochem 1983; 52 : 301-54.

4. Weiss R. A., Teich N. M., Varmus H. E., Coffin J. M. RNA tumor viruses. Cold Spring Harbor, New York: Cold Spring Harbor Laboratory, 1982.

\section{ADRESSE}

D. Stçhelin : Inserm (U I 86). Institut Pasteur, 15 , rue Camille Guérin,59019 Lille.

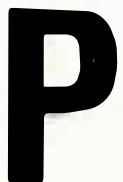

lusieurs agents ont été reconnus capables d'engendrer des cancers : virus, radiations ionisantes, cancérogènes chimiques, hormones, etc. Si les virus constituent un apport de matériel génétique extérieur à la cellule, les autres agents induisent des modifications durables du patrimoine génétique propre à la cellule, et tout indique que dans ce cas plusieurs modifications sont nécessaires avant que la cellule ne devienne cancéreuse. Or chaque cellule de notre organisme contient tout notre patrimoine génétique : environ 50000 gènes dans l'ADN de nos chromosomes sont responsables de notre programmation biologique. Ainsi l'ovule fécondé (une seule cellule à l'origine) utilise des modules de ce programme pour se multiplier (un adulte possède quelque 60000 milliards de cellules), pour se différencier (on dénombre quelque 200 tissus différents dans notre organisme), ou encore pour entretenir le fonctionnement des cellules spécialisées formées. Il est alors tentant de spéculer que certains gènes de ces programmes pourraient se mettre à fonctionner anormalement, et engendreraient ainsi dans certains cas la formation de cancers. Mais alors, comment identifier parmi nos 50000 gènes ceux qui pourraient devenir les coupables? C'est aux virus que l'on doit une véritable révolution dans l'approche moléculaire des cancers.

-En I9I I, l'anglais P. Rous isolait un virus capable d'engendrer des tumeurs du tissu conjonctif (sarco- mes) chez le poulet, en quelques semaines. Par la suite, plus d'une vingtaine d'autres virus du même genre (les rétrovirus des cancers aigus) furent isolés chez divers animaux (poulet, souris, chat, singe, etc.). Ils engendraient des types spécifiques de cancers : sarcomes, leucémies aiguës, carcinomes. Ces virus bien connus aujourd'hui possèdent deux caractéristiques remarquables : d'une part, leur matériel génétique (génome) est simple (seulement trois ou quatre gènes) et d'autre part, leur génome d'ARN utilise une enzyme particulière (transcriptase réverse ou rétropolymérase) pour se copier en $\mathrm{ADN}$ (d'où leur nom de "rétrovirus ") et s'intégrer alors stablement dans les chromosomes de la cellule infectée. Ces parfaits parasites détournent alors jusqu'à I \% de la machinerie cellulaire à leur profit pour fabriquer les virions qui iront à leur tour infecter d'autres cellules.

\section{Les oncogènes}

L'avènement des techniques de biologie moléculaire et du génie génétique a permis rapidement de montrer que ces rétrovirus contenaient des gènes de cancers ou oncogènes, dont j'eus le privilège d'isoler le premier en $1976[\mathrm{I}$ ], appelé gène src (pour sarcomes) chez le virus isolé par P. Rous. A notre surprise, nous montrions la même année que des cellules normales contenaient un gène semblable (appelé $c$-src ou src cellulaire), non seulement chez le poulet, mais encore chez toutes les 


\begin{tabular}{|c|c|c|}
\hline \multicolumn{3}{|c|}{ Tableau 1} \\
\hline $\begin{array}{l}\text { chromosome humain } \\
\text { numéro, région }\end{array}$ & $\begin{array}{l}\text { oncogène } \\
\text { cellulaire }\end{array}$ & $\begin{array}{c}\text { implication probable } \\
\text { dans } \\
\text { les cancers humains }\end{array}$ \\
\hline $\begin{array}{c}1 \text { p32 } \\
1 \text { p11-p13 } \\
1 \text { q11-q13 } \\
2 \text { p23-p24 } \\
3 \text { p25 } \\
5 \text { q34 } \\
6 \text { q15-q24 } \\
7 \text { pter-q22 } \\
8 \text { q22 } \\
8 \text { q24 } \\
9 \text { q34 } \\
11 \text { pter-p15. } 1 \\
11 \text { q23-q24 } \\
12 \text { pter-p12 } \\
14 \text { q21-q31 } \\
15 \text { q26.1 } \\
17 \text { q21-q22 } \\
20 \text { q12-q13 } \\
22 \text { q13.1 }\end{array}$ & $\begin{array}{l}\text { B-lym } \\
N \text {-ras } \\
\text { ski } \\
N \text {-myc } \\
\text { raf/mil } \\
\text { fms } \\
\text { myb } \\
\text { erbB } \\
\text { mos } \\
\text { myc } \\
\text { abl } \\
\text { H-ras/bas } \\
\text { ets } \\
\text { K-ras } \\
\text { fos } \\
\text { fes } \\
\text { erbA } \\
\text { src } \\
\text { sis/fis }\end{array}$ & $\begin{array}{c}\text { lymphomes } \\
\text { neuroblastomes } \\
\text { neuroblastomes } \\
\text { leucémies } \\
\text { tumeurs de Burkitt } \\
\text { leucémies? } \\
\text { carcinomes } \\
\text { carcinomes }\end{array}$ \\
\hline
\end{tabular}

espèces animales vertébrées testées [2]. Ces résultats furent repris dans de nombreux laboratoires et étendus à d'autres rétrovirus. Ainsi une vingtaine d'oncogènes différents furent découverts et caractérisés $[3,4]$ (tabl. I).

La découverte des oncogènes permet donc d'élaborer une théorie unificatrice du cancer : les cellules normales contiennent des gènes très semblables à ceux trouvés chez des virus hautement cancérogènes. De tels virus détournent ces gènes cellulaires à leur profit pour les réintroduire, sous leur propre contrôle, dans les nouvelles cellules qu'ils infectent ( $f i g .1)$ : ils ne sont qu'un cas particulier d'activation anormale de ces gènes cellulaires. D'autres agents (cancérogènes chimiques, aberrations chromosomiques, rayonnements, etc.) ont peut-être pour cible ces gènes cellulaires.

Figure 1. Formation des rétrovirus cancérogènes et modèle de cancérogénèse virale.

(1) Infection d'une cellule normale par un rétrovirus non transformant.

(2) Transduction par ce rétrovirus d'un gène c-onc devenant ainsi v-onc dès qu'il est intégré dans le génome viral.

(3) Transformation d'une cellule par le rétrovirus oncogène formé en" (2); le gène v-onc, souvent qualitativement modifié au cours de l'évolution du virus et placé sous le contrôle des séquences stimulatrices $d u$ LTR viral, entraîne la cancérisation.

Gène Gag = gène viral codant pour une protéine virale.

Gène Pol=gène viral codant pour la transcriptase reverse (ou rétropolymérase).

Gène Env=gène viral codant pour une protéine d'enveloppe de la particule virale.

$L T R=$ Long terminal repeat, séquences situées de part et d'autre du virus correspondant au site d'intégration dans r ADN cellulaire et contenant notamment les " séquences stimulatrices "virales, stimulant la transcription des gènes viraux.

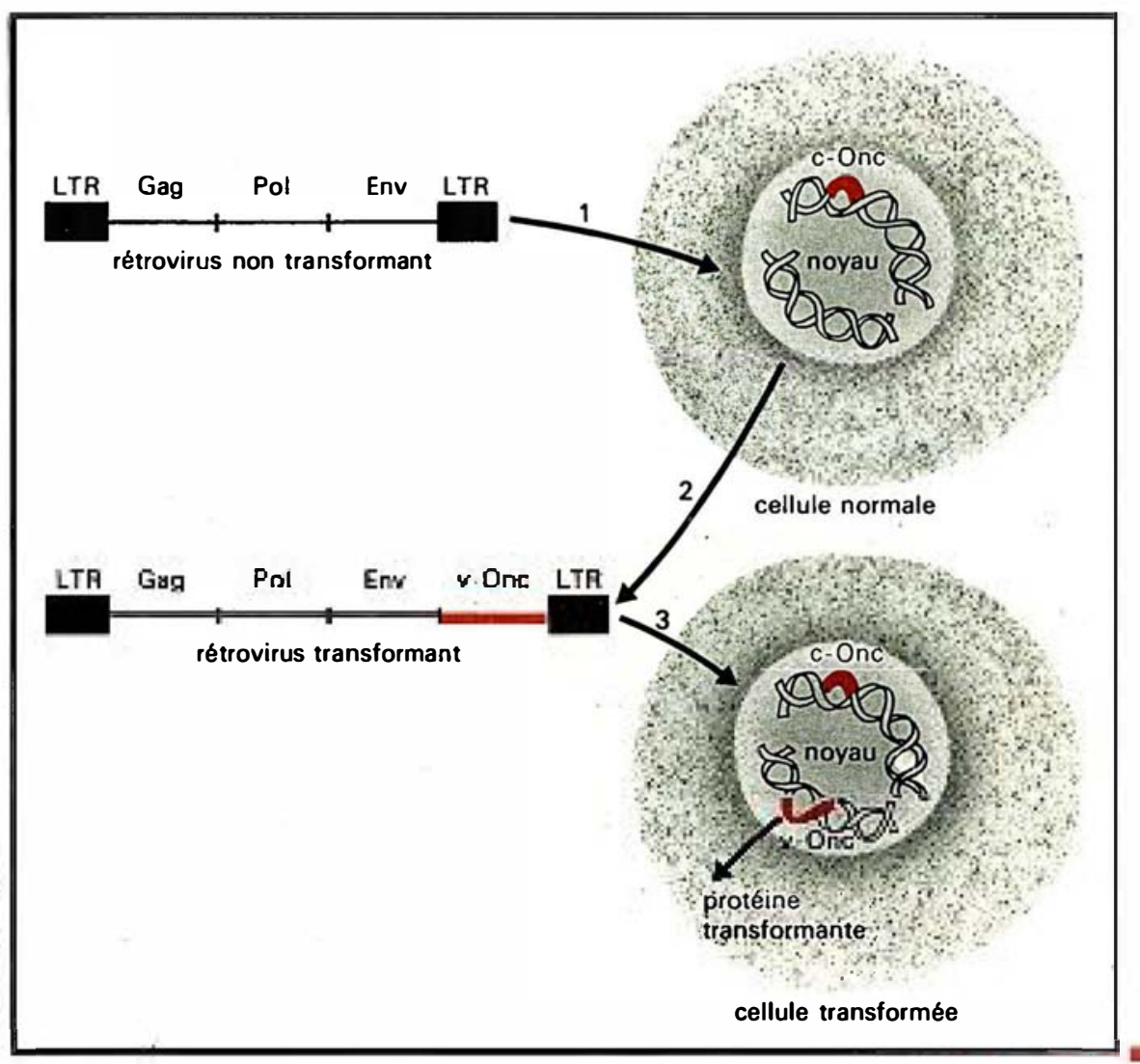




\section{REFERENCES}

5. Ellis R. W., Defeo D., Furth M. E., Scolnick E. M. Mouse cells contain two distinct ras gene mRNA species that can be translated into a p21onc protein. Mol Cell Biol 1982; 2 : 1339-45.

6. Tabin C. J., Bradley S. M., Bargman C. I. et al. Mechanism of activation of a human oncogene. Nature 1982; 300: 143-9.

7. Reddy E. P., Reynolds R. K., Santos E., Barbacid A. A joint mutation is responsible for the acquisition of transforming properties by the T-24 human bladder carcinoma oncogene. Nature 1982; 300: 149-52.

8. Der C., Krontiris T., Cooper G. M. Transforming genes of human bladder and lung carcinoma lines are homologous to the ras genes of Harvey and Kirsten sarcoma viruses. Proc Natl Aca Sci USA 1982; 79: 3637-40.

9. Land H., Parada L. F., Weinberg R. A. Tumorogenic conversion of primary embryo fibroblasts requires at least two cooperating oncogenes. Nature 1983; 304: 596-602.

ro. Roussel M., Saule S., Logrou C. et al. Three new types of viral oncogene of cellular origin specific for haematopoietic cell transformation. Nature 1979; 281 : 452-5

I I. Frykberg L., Palmieri S., Beug H., Graf T., Hayman M. J., Vennstrom B. Transforming capacities of avian erythroblastosis virus mutants deleted in the erbA or erbB oncogenes. Cell 1983 ; 32 : 227-38.

12. Debuire B., Henry C., Benaissa M. et al. Sequencing the erbA gene of avian erythroblastosis virus reveals a new type of oncogene. Science 1984; $224: 1456-9$.

13. Graf T., Stéhelin D. Avian leukemic viruses, oncogenes and genome structure. Biochim Biophys Acta 1982; 651 : 245-71.

14. Coll J., Righi M., de Taisne C., Dissous C., Gegonne A., Stéhelin D. Molecular cloning of the avian acute transforming retrovirus $\mathrm{MH} 2$ reveals a novel cell, derived sequence ( $v$-mil) in addition to the myc oncogene. $E m b o$ J $1983 ; 2: 2189-94$.

15. Galibert F., Dupont de Dinechin S., Righi M., Stéhelin D. The second oncogene mil of avian retrovirus $\mathrm{MH}_{2}$ is related to the src gene
D'autre part, que ces oncogènes cellulaires se soient maintenus lors de la phylogenèse des espèces, valide les systèmes animaux et justifie leur recours pour. découvrir des oncogènes potentiels que l'on peut alors rapidement identifier chez l'homme en utilisant le génie génétique. Le tableau I indique ainsi la localisation de plusieurs de ces gènes cellulaires dans les chromosomes humains normaux.

Enfin, cette théorie suggère que des cancers de types cellulaires différents pourraient en fait être initiés par le dérèglement des mêmes oncogènes, ou au contraire, que des cancers de même type cellulaire pourraient être initiés par le dérèglement d'oncogènes différents.

Aussi fécondes que paraissaient ces découvertes, il restait à montrer que des oncogènes cellulaires pouvaient être impliqués dans des cancers naturels chez l'homme. Des recherches récentes viennent de démontrer deux exemples d'une telle implication intéressant les oncogènes myc (dont il est question dans un autre article de ce numéro) et ras.

\section{Le gène ras}

Cet oncogène fut découvert il y a plusieurs années dans un rétrovirus murin [5]. Mais il fut retrouvé indépendamment dans une autre approche des gènes de cancer que nous allons décrire brièvement (fig. 2). Certaines cellules fibroblastiques de souris (appelées $\mathrm{NIH}_{3} \mathrm{~T}_{3}$ ) possèdent la propriété d'accepter un ADN étranger. Ces cellules ne sont plus tout à fait normales : elles semblent se trouver à un stade de précancérisation (dont le mécanisme n'est pas encore connu) et peuvent être maintenues en culture in vitro. Si l'on extrait l'ADN d'un tissu sain humain, et qu'on l'introduit dans ces cellules révélatrices de souris (c'est une transfection), on ne constate pas de modification morphologique décelable (fig. 2). En revanche, la même expérience réalisée avec l'ADN de certaines tumeurs humaines de la vessie (mais aussi du poumon, du colon, de neuroblastomes, etc.) modifie les cellules de souris $\mathrm{NIH}_{3} \mathrm{~T}_{3}$ et les transforme, c'est-à-dire leur donne l'aspect de cellules cancéreuses qui crosssent anarchiquement. Dans la tumeur étudiée, le gène cellulaire humain a été isolé par génie génétique : il s'agit d'un gène cellulaire $c$-ras, correspondant à l'oncogène viral ras précité, isolé indépendamment. L'analyse fine de ce gène a montré qu'une seule mutation bien précise, modifiant le douzième acide aminé à partir de l'extrémité $\mathrm{N}$-terminale de la protéine $c$-ras était responsable [6-8] de la transformation des cellules $\mathrm{NIH}_{3} \mathrm{~T}_{3}$. Ceci a été prouvé par l'expérience croisée suivantes : si on prend le gène $c$-ras humain normal et qu'on modifie artificiellement la région correspondant au douzième acide aminé, ce gène devient transformant sur les cellules $\mathrm{NIH}_{3} \mathrm{~T}_{3}$ révélatrices; inversement si l'on prend le gène $c$-ras de la tumeur humaine et qu'on le modifie pour que le douzième acide soit comme dans le gène normal, ce gène ne transforme plus les cellules révélatrices. Notons ici que cet effet n'est pas observé sur des fibroblastes sains de rongeurs, mais uniquement sur des cellules révélatrices déjà précancéreuses.

De ces expériences, nous concluons que dans certains cancers de la vessie, le gène humain $c$-ras est activé par une mutation bien précise qui modifie son produit, et le rend transformant sur les cellules révélatrices $\mathrm{NIH}_{3} \mathrm{~T}_{3}$.

\section{dans la cancérisation}

Nous avons dit plus haut que les cancers naturels impliquaient plusieurs étapes. Peut-on relever le défi de décrypter ces différentes étapes au niveau expérimental? Dans l'exemple qui vient d'être décrit, il convient de remarquer que l'on se plaçait dans des conditions un peu particulières : les cellules révélatrices étaient déjà partiellement anormales; quelque chose s'était passé dans ces cellules, qui les prédisposait à se transformer sous l'action du gène cellulaire ras activé de la tumeur de vessie. Ce test ne marchait pas avec des cellules révélatrices normales. L'hypothèse de la collaboration de plusieurs oncogènes dans la transformation cancéreuse a été confirmée par des expé- 


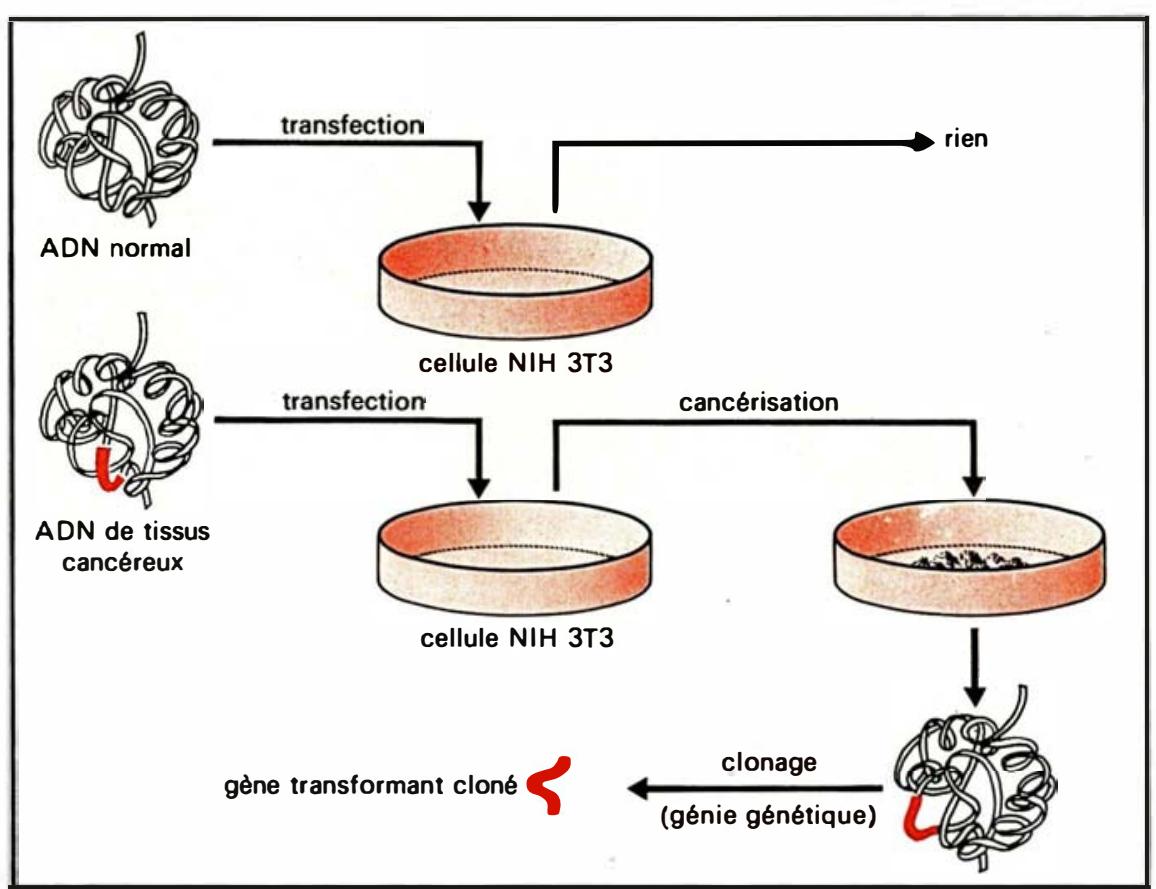

Figure 2. Test de l'ADN de tumeurs

riences de reconstitution par transfection des gènes activés ras et/ou myc sur des fibroblastes d'embryons de rat [9] (donc des cellules révélatrices normales et non pas des $\mathrm{NIH}_{3} \mathrm{~T}_{3}$ précancéreuses) : le gène myc seul n'avait pas d'effet transformant (mais il agit peut-être en allongeant la durée de vie des cellules). Le gène ras avait un effet sur quelques cellules, mais ces cellules modifiées redevenaient rapidement normales et n'étaient pas tumorigènes après greffe chez la souris nude. En revanche les fibroblastes transfectés à la fois par ras et myc activés se transformaient et étaient tumorigènes chez la souris nude (les tumeurs régressant toutefois au bout de quelques semaines, donc ne tuant pas l'animal).

Les gènes activés ras et myc agissaient donc bien de concert pour

transformer les fibroblastes de rat, et il devenait possible de définir des systèmes expérimentaux distinguant deux étapes dans la transformation. Le fait que les fibroblastes transformés étaient tumorigènes impliquait la synergie/complémentation des deux gènes. Le fait qu'ils formaient des tumeurs régressives chez la souris nude suggérait qu'un troisième gène (ou plus) était peutêtre nécessaire pour engendrer des tumeurs ne régressant plus (et peut-être un autre encore pour induire des métastases).

D'autres études menées indépendamment, en particulier dans notre laboratoire, devaient aboutir à la même conclusion que deux oncogènes pouvaient agir en synergie/complémentation. En effet, certains rétrovirus chez l'oiseau contenaient non pas un, mais deux oncogènes distincts. Ces doubles oncogènes n'étaient pas rassemblés par hasard : des travaux impliquant une fois de plus le génie génétique permirent d'inactiver l'un ou l'autre oncogène de ces virus, pour étudier leur rôle dans le pouvoir transformant. Nous présentons ici deux exemples de résultats obtenus a vec des virus à doubles oncogènes.

\section{à doubles oncogènes}

Le rétrovirus de l'érythroblastose aviaire $(\mathrm{AEV})$ : ce virus contient les deux oncogènes erbA et erbB [1012]. Le gène erbB induit une transformation des érythroblastes de poulet, qui ne se différencient plus correctement. Cette leucémie érythroïle chronique peut régresser, mais plus généralement elle finit par tuer l'animal au bout de quelques semaines. Le gène erbA seul n'induit pas de cancers. En revanche le virus contenant les deux oncogènes erbA et erbB induit une leucémie érythroïde aiguë qui tue l'animal en une vingtaine de jours. L'examen sanguin révèle que les cellules érythroïdes de l'animal sont bloquées à un stade immature de leur différenciation où elles se multiplient activement. Nous déduisons de ces résultats que le gène erbA potentialise l'action du gène erbB dans la cancérisation des cellules érythroïdes du poulet.
Le rétrovirus $\mathrm{MH}_{2}$ : ce virus contient les gènes myc et mil [13I5]. L'oncogène myc entraîne la formation de sarcomes, carcinomes (rein, foie) ou transforme les macrophages (donc trois types cellulaires différents sont touchés). Mais ces cancérisations sont inefficaces (seuls de rares poulets les subissent) et les cancers mettent plusieurs mois pour se développer. Le gène mil ne semble pas doué d'un potentiel oncogène important (encore que les expériences décisives en la matière ne soient pas terminées). En revanche, le virus contenant les gènes myc et mil produit des sarcomes et carcinomes dans tous les poulets inoculés; ces tumeurs sont détectables en moins de 15 jours et provoquent rapidement la mort des poulets. Nous concluons donc ici encore que deux 
oncogènes distincts peuvent agir en synergie/complémentation dans la formation de tumeurs.

Il existe donc aujourd'hui une théorie unificatrice concernant la formation des cancers : certains gènes cellulaires remplissant des fonctions normales importantes dans notre physiologie peuvent dans des circonstances exceptionnelles échapper aux contrôles dont ils font l'objet. De tels gènes peuvent alors devenir des oncogènes actifs et entrainer la formation de cancers. Une vingtaine d'oncogènes cellulaires a été isolée. On ne sait pas combien il en existe dans nos cellules, mais il paraît raisonnable de penser que la liste ne comprendra pas plus de quelques dizaines de tels gènes.

Tous les oncogènes viraux ont été impliqués dans des cancers animaux. Leurs homologues cellulaires ont été impliqués dans des cancers non viraux, à la fois chez l'animal et, pour deux au moins d'entre eux, chez l'homme.

Il s'en suit que les'cancers chez l'animal et chez l'homme mettent probablement en jeu des mécanismes fondamentaux semblables. Le voile se lève doucement sur certains de ces mécanismes et des systèmes expérimentaux commencent à apparaître, qui permettent de tester l'activité d'un oncogène donné, ou même d'étudier l'interaction de couples d'oncogènes. Apparaît ainsi le concept, surprenant pour beaucoup, que plusieurs oncogènes distincts peuvent initier la cancérisation d'un même type de tissus, et que réciproquement un même oncogène peut intervenir dans la cancérisation de plusieurs types tissulaires différents. Il est encore trop tôt pour mesurer l'impact concret de ces découvertes du point de vue clinique.

On peut cependant espérer qu'apparaîtront progressivement pour certains cancers des techniques permettant une approche nosologique cohérente, des tests fiables susceptibles d'orienter l'approche thérapeutique, et peut-être un jour des thérapies spécifiques touchant des cellules cancéreuses données, sans

\section{TIRES A PART}

D. Stéhelin : Inserm (U I86). Institut Pasteur, être traumatisantes pour les cellules normales ni pour l'individu concerné

\section{Summary}

Viral oncogenes ( $v$-onc) of retroviruses include at least one cellular equivalent, called c-onc, which is the precursor of $v$-onc genes. This phenomenon results from a DNA recombination between viral and cell genomes. Cellular oncogenes have probably fundamental functions in regulation of cell cycle and proliferation; they are transcriptionnally active in growing cells and during embryonic development.

Transformation of a $c$-onc gene into a transforming gene results from its qualitative modification or from changes in its expression.

Cancerogenesis is most likely a complex process involving the activation of several oncogenes. 